\title{
Bajo la experiencia del exilio y el exilio como experiencia
}

\author{
Entrevista con Antolín Sánchez Cuervo', \\ por Camila Díaz Parraguez ${ }^{2}$
}

\section{Introducción}

Los días 28 y 29 de septiembre de 2017, tuvo lugar el Primer Seminario Internacional sobre el exilio, llamado La experiencia del exilio y el exilio como experiencia ${ }^{3}$, realizado por la Escuela de Filosofía de la Universidad Católica Silva Henríquez, y por el Centro de Estudios Avanzados de la Universidad de Playa Ancha. En este encuentro participaron como expositores, entre otros destacados académicos, Adriana Arpini, de la Universidad Nacional de Cuyo, Argentina; Patricia Bonzi y Loreto Rebolledo, de la Universidad de Chile; Cristina Hurtado y Cecilia Sánchez, de la Universidad Academia de Humanismo Cristiano, Chile; José Francisco Martin, de la Universidad de Torino, Italia, y Antolín Sánchez Cuervo, del Consejo Superior de Investigaciones Científicas de Madrid, España. Aprovechando la presencia de este último con la conferencia inaugural "El exilio intelectual español de 1939 en perspectiva transnacional", se realizó la entrevista que compartimos a continuación.

\footnotetext{
1 Antolín Sánchez Cuervo es licenciado y doctor en Filosofía por la Universidad Pontificia Comillas de Madrid. Actualmente es investigador del Instituto de Filosofía del CSIC (Consejo Superior de Investigaciones Científicas). Sus líneas de investigación se enmarcan en la Filosofía Iberoamericana, en la historia y la memoria, en el exilio, especialmente en el Exilio Republicano español del '39, y en la crítica de la cultura. 2 Camila Díaz Parraguez es licenciada en Educación y profesora de Pedagogía en Filosofía por la Universidad Católica Silva Henríquez.

3 Este encuentro fue auspiciado por el Fondecyt Postdoctoral № 3160779 "Narrativas marginales de/sobre Valparaíso: una mirada analítico-crítica a los imaginarios de resistencia a la modernización 1925-1980", del Dr. Braulio Rojas, y por el Fondecyt de Iniciación N 11160148 "Re-pensar el exilio: ampliaciones de sentido para un análisis filosófico en clave latinoamericana", de la Dra. Mariela Avila.
} 
Quisiera agradecer la instancia académica para llevar a cabo esta entrevista, en primer lugar a la profesora Mariela Avila, por brindarme el espacio y confiar en mi trabajo y, en segundo lugar, al profesor Antolín Sánchez Cuervo, por su disposición y apertura en este diálogo, que pretende dar a conocer sus aportes, conocimientos y experiencias en el mundo de la filosofía.

Camila Díaz: Aprovechando su estadía en estas tierras, antes de adentrarnos de lleno en la entrevista, quisiera agradecer en nombre propio y de la revista Hermenéutica Intercultural esta posibilidad de diálogo, para el que usted ha brindado su total disposición, tanto para participar, como para darnos a conocer sus aportes en el mundo de la filosofía.

Antolín Sánchez: Pues de nada, muchas gracias, un placer.

C.D: Así, quiero dar inicio con una primera pregunta, que dice relación con sus líneas de investigación. Tengo entendido que sus líneas de trabajo, por una parte se relacionan con la filosofía iberoamericana, la historia, la memoria, el exilio y la crítica de la cultura, entonces, ¿cómo surge el interés por estas temáticas? $\mathrm{O}$, dicho de otro modo, ¿qué fue lo que gatilló su interés? Finalmente, ¿existe alguna preocupación o experiencia personal que lo haya llevado a trabajar estos problemas?

A.S: Sí, sí, yo creo que, como siempre sucede con el interés teórico, en mi caso hubo una mediación personal, experiencial. Es un interés que no surgió de la nada. Surgió a partir de mi experiencia en México ya que, debido a circunstancias personales, visité México, y a raíz de eso me interesé por la filosofía mexicana e iberoamericana en general, y en particular por el mundo del Exilio Republicano español de 1939 en México. Más en concreto, me interesa su legado cultural y filosófico que, además, era y sigue siendo una temática relativamente novedosa, que aún necesita mucha exploración y mucho trabajo para ser rescatada. Esto fue lo que me "enganchó" con estos problemas, pero, como sucede en estos casos y en estas cosas, hubo una circunstancia personal, biográfica que hizo de mediación.

C.D: Veo que son muchos los temas que aborda en su trabajo, yo particularmente quedé impresionada con su curriculum, tengo que decirlo. 
A.S: Bueno, no es para tanto, tengo una cierta edad.

C.D: Me parece impresionante la cantidad de publicaciones y participaciones que tiene en distintos países de Latinoamérica y Europa. En relación a sus participaciones, particularmente en Chile, como la de diciembre del 2014 en Talca, durante la IV Jornada de Estudios sobre Ideas, donde presentó el trabajo "El debate entre la historia y la memoria y su proyección en la historia de las ideas en América Latina" como conferencia inaugural; o su participación de 2012 en Santiago de Chile, durante el IV Congreso Iberoamericano de Filosofía, me gustaría saber: ¿qué impresiones tiene, en primer lugar, de sus estancias académicas en Chile? Y, en segundo lugar, ¿cree que de un tiempo a esta parte hay un mayor interés en las escuelas de filosofía por abordar las temáticas que usted trabaja? Ejemplo de ello podría ser su última participación en el seminario La experiencia del exilio y el exilio como experiencia, realizado entre el 28 y el 29 de septiembre de 2017.

A.S: ¿Interés en las escuelas de filosofía en Chile o en general?

C.D: Particularmente en el caso de Chile.

A.S: Bueno, Chile es un país que desgraciadamente conozco poco, que espero conocer mucho mejor aún, y al que espero venir muchas más veces, porque para mí siempre es una experiencia muy grata y muy enriquecedora. Además de lo bien tratado que soy siempre aquí, y América Latina en general, es una realidad tremendamente heterogénea y diversa, ya que no es lo mismo México, lugar que sí conozco bastante bien, que Chile, o que Colombia. Entonces, aquí en Chile noto que es una Latinoamérica diferente.

C.D: ¿En qué sentido diferente? Si pudiera profundizar un poco esa idea.

A.S: Bueno, en principio la historia es diferente, los problemas, aunque haya aires de familia en relación con México, por ejemplo, incluso en la geografía. A mí Chile siempre me ha parecido una especie de isla, me siento como en una isla entre los Andes, el Océano Pacifico, el hielo y el desierto. Santiago de Chile es una ciudad que tiene muy poco que ver con, por ejemplo, Ciudad de México, aunque se hable el mismo 
idioma, y se habla el mismo idioma con modismos muy diferentes además. Aunque apenas he venido cuatro o cinco veces cada vez que vengo, procuro empaparme lo más posible, hacer lecturas, conocer un poco mejor este país. Conozco un poco Santiago de Chile y Valparaíso, y aunque estuve en una ocasión también en Talca, siempre me muevo entre Santiago y Valparaíso, que es una ciudad que me parece muy fascinante, por su carácter porteño, su apertura al mar, con todo lo que ello implica. Académicamente hablando, tengo la impresión de que en Chile la academia, la universidad, está un tanto fragmentada, me da un poquito esa impresión, de que las actividades académicas, científicas, por lo menos en el ámbito de la filosofía, están muy fragmentadas, y no sé si eso pueda ser una relativa dificultad. Lo que percibo es un interés creciente por la filosofía latinoamericana, que creo que eso es algo común al resto de América Latina. Pienso que en México sucede algo parecido, en Argentina también, respecto de la filosofía española. En España, por ejemplo, hay una tremenda ignorancia respecto a la filosofía latinoamericana, pero hay un interés creciente por la filosofía española, lo cual me parece interesante y prometedor, ya que creo es algo necesario de potenciar, ese interés por las propias tradiciones filosóficas, por la manera en que se han recibido y metabolizado las tendencias filosóficas ajenas y hegemónicas. Siempre en España, en el Instituto de Filosofía del $\mathrm{CSIC}^{4}$, estoy muy vinculado a los temas que tienen que ver con pensar en español, con la Enciclopedia lberoamericana de Filosofía. El Congreso al que tú aludiste fue uno de los promocionados por dicha Enciclopedia. Entonces, esa es una temática en la que algunos estamos embarcados desde hace tiempo, y en la que yo percibo un entusiasmo creciente o moderadamente creciente.

C.D: Entonces, en resumen, tendría buenas experiencias respecto a sus participaciones en Chile.

A.S: Sí, sí, a mí me parece que además de ese interés creciente, el nivel académico es muy bueno. Esto lo pienso para mis adentros,

4 El Consejo Superior de Investigaciones Científicas es la mayor institución pública dedicada a la investigación en España. Información disponible en http://www.csic.es 
porque yo no soy quien para juzgar el nivel académico de este país o del otro que visito, y que conozco apenas, pero yo me siento a gusto, cómodo, y percibo que hay discusiones muy interesantes. Creo que hay también un interés creciente, aunque quizás lento, porque necesita su tiempo, por aquellos temas relacionados con la memoria, con el pasado, pues Chile tiene un pasado reciente traumático como el de otros países, como el de Argentina por supuesto, como el de España, y creo que eso marca, pero que es un camino necesario. El camino de la memoria crítica, de la memoria política, de pensar el propio pasado, es fundamental para transformar el presente.

C.D: ¿Es la investigación su mayor interés o lo es la docencia? Pregunto porque veo que gran parte de su trabajo se enmarca en la investigación, más que en la docencia. Entonces, ¿son ambas áreas compatibles o tiene alguna preferencia?

A.S: Son compatibles, deberían serlo siempre. Creo que las dos se enriquecen mutuamente, pienso que a todo investigador le gusta o debería gustarle la docencia, y que todo docente debería investigar también. En mi caso, mi perfil profesional me condiciona mucho, yo soy investigador del Estado, pertenezco a un organismo científico del Estado, en el que los investigadores únicamente nos dedicamos a la investigación, y donde no se imparte docencia. Esto último muchos investigadores lo echamos de menos a veces, por lo que antes comentaba, porque las dos actividades se retroalimentan. Sin embargo, aunque no se imparta docencia en el Consejo Superior de Investigaciones Científicas, CSIC, procuramos siempre estar en contacto con el mundo universitario, impartimos muchos seminarios, cursos de posgrado, constantemente nos invitan a colaborar en programas de doctorado y de maestría, para dar clases, asignaturas, etc. En fin, nos preocupa mucho ese vínculo, el puente con el mundo universitario, y procuramos no descuidarlo, lo que pasa es que, inevitablemente, el hecho de que no se imparta docencia en el lugar de trabajo condiciona el curriculum, por lo que habrás visto en mi curriculum, muchas conferencias, seminarios, publicaciones y poca docencia, o más bien puntual. 
C.D: En ese sentido, se podría decir que no es una cuestión de preferencia, sino más bien cuestiones fortuitas.

A.S: Bueno, es que ni siquiera puedo elegir (risas), porque el trabajo que tengo que hacer, con el que yo estoy comprometido, es el de la investigación, entonces, de entrada y por definición, no puedo elegir, soy investigador. Sin embargo, desde ese perfil estoy a favor de abrir todos los puentes posibles con el mundo universitario, y cuando tengo la ocasión de impartir algún curso para mí es una experiencia enriquecedora, sin duda.

C.D: Mi siguiente pregunta dice relación con la filosofía y su experiencia. Teniendo en consideración que la filosofía es una disciplina que siempre deja más interrogantes que respuestas, por su carácter crítico y reflexivo, ¿cree usted que ha sido la filosofía su vocación o es quizás una cuestión fortuita? ¿Podría comentar sus inicios en la filosofía?

A.S: Bueno, para responder con mínima profundidad a tu pregunta necesitaría una sesión de psicoanálisis.

C.D: (Risas) No es una pregunta fácil de responder.

A.S: La vocación personal siempre es algo que anida en lo profundo de uno, que a veces es oscuro, difuso; entonces, creo que hasta que me muera tendré interrogantes relacionados con mi identidad profunda, mi vocación más profunda y por lo tanto oscura. La filosofía es una vocación con la que me siento a gusto, aunque unas veces se tienen más satisfacciones que otras, inevitablemente. La vocación filosófica está muy torpedeada por la creciente burocratización de la academia, tanto en el ámbito de la docencia como en el de la investigación, y eso quizás porque la vocación de la filosofía está orientada a poner todo en cuestión, es tremendamente, como tu bien mencionaste, crítica y autocrítica, reflexiva; es una vocación orientada no tanto a la búsqueda de respuestas como al replanteamiento de preguntas que nunca tienen respuestas definitivas, pero que son indispensables para vivir humanamente. Entonces esa vocación me llama la atención desde que era muy joven, aunque hay momentos de debilidad, como por ejemplo cuando tenemos que luchar contra las burocracias, contra el 
costo de esta estupidez de los índices de impacto, en fin, todas estas políticas científicas tan antivocacionales.

C.D: Pensando desde la experiencia, en mi caso, la filosofía no era la primera opción, aunque siempre fue pedagogía, quería estudiar educación física.

A.S: Bueno, la filosofía tiene la virtud de que se puede relacionar y conectar con todo, y por supuesto, con la educación física también.

C.D: Vamos comentando experiencias, porque me llama la atención el modo en que se llega a la filosofía, ya que no todos lo hacen de la misma manera. Para concluir, quisiera saber cuáles son sus proyecciones, si existen nuevos textos o proyectos, y si habrá próximas visitas a nuestro país. Y finalmente, si tuviera que dar un consejo a las nuevas generaciones que se inician en el mundo de la filosofía, ¿cuál sería?

A.S: Son varias preguntas. Primero, sí, espero venir, tengo expectativas porque me encantaría, y aunque eso siempre depende de muchos factores, deseo nunca me va a faltar. Respecto de las expectativas, en un sentido más convencional y académico, siempre hay proyectos de libros por escribir, de tareas colectivas que coordinar. Esas tareas forman parte de proyectos de investigación que, una vez que conseguimos, hay que coordinar. Un proyecto implica coordinación, mover todos sus engranajes, y ahí se van madurando. En concreto, tengo en la cabeza una trilogía sobre el legado filosófico del Exilio Republicano español del '39, que nunca he podido sentarme a escribir por culpa de las tareas burocráticas de la gestión, entre otras cosas. Eso es lo que me gustaría hacer en los próximos dos, tres, cuatro años. Por otra parte, en este momento yo soy investigador principal, que es el término que en España se emplea para referirse a la persona que coordina un proyecto del Plan Nacional, que dura cuatro años; entonces, ir coordinando este proyecto es también mi aspiración a corto plazo. Te estoy hablando de pequeñas miserias, pero en un sentido más ambicioso, y quizás también un poco más vago, me gustaría sacar la investigación filosófica, en la medida de lo posible, de estas sinergias a veces empobrecedoras, tan condicionadas y restringidas por los aparatos burocráticos, por la gestión, por la imposición de 
los criterios para publicar, por los índices de impacto, etc., y en este sentido ser más creativos. Me gustaría dar un impulso creativo a la investigación filosófica, sin que por ello deje de ser rigurosa, es decir, sacarla de la academia sin abandonarla: que pusiera un pie fuera para buscar puentes con la sociedad civil y sus iniciativas. Y también con otras iniciativas de muy diverso perfil, que pueden ser museos, teatros o actividades de tipo social, o reivindicaciones políticas. Creo que la filosofía sirve, o debe servir también, para llegar a esos espacios.

C.D: ¿Y el consejo a la nuevas generaciones que se inician en el mundo de la filosofía?

A.S: Lo de dar consejo es algo a lo que he rehuido siempre, porque me da miedo caer en actitudes paternalistas, yo puedo hacer sugerencias.

C.D: Hablemos entonces de sugerencias.

A.S: Alguna que otra sugerencia a quien se quiera dedicar a esto, sería que se asegure previamente que tiene una vocación muy fuerte para ello, porque el futuro no es fácil, al menos el futuro profesional. Vivir de la filosofía es muy complicado y exige un deseo y un amor a la filosofía muy grandes, una vocación comprometedora. Que esté muy seguro, y si lo está que disfrute del camino, pues si se tiene vocación el camino es muy grato y enriquecedor, como una aventura. Realmente, la vocación por la filosofía es una vocación de aventura y eso también es muy gratificante, es como lanzarse al océano en barco y ver hasta donde se llega.

C.D: Se podría decir que usted es feliz con la filosofía.

A.S: Eso de ser feliz en un mundo tan calamitoso es algo complicado (risas), pero tampoco se puede uno ahogar en un mar de pesimismos. Creo que es importante mantener un equilibrio, hay que ser realistas. Hablábamos antes de la memoria crítica, la memoria política, de la cultura de la memoria que, además, es algo que está en alza a nivel global en los últimos años. Para la filosofía es muy importante la memoria de las víctimas y asumir esa memoria significa asumir la experiencia del duelo y de la deuda en la propia vida. Entonces, tampoco podemos andar con optimismos frívolos y fáciles, pero sí con una afirmación de 
la propia vocación de la profesión, con un mínimo de optimismo para luchar, para encauzar esa voluntad de aventura y ser feliz, aunque sea un término que me intimida un poco, pero, por lo menos, estar un poco contentos, satisfechos y con ganas de trabajar.

C.D: Profesor, nuevamente le doy las gracias por esta instancia de intercambio alrededor de la filosofía y de la propia experiencia filosófica.

A.S: De nada, gracias a ti. Ha sido una entrevista muy amable y muy noble. 